\title{
La Voce dei Pazienti
}

\section{Storia di una brutta notizia e di un grande coraggio: l'esempio di Chiara}

Questa storia ha dentro di sé l'essenza stessa della vita con i suoi alti e bassi, con i colpi avversi della fortuna e con la reazione delle migliori risorse che sono dentro di noi. Con il cuore in mano Chiara, una manager con una vita familiare che fino a quel momento si poteva definire idilliaca, ci racconta il suo percorso: dalla scoperta di una patologia importante all'incontro con le sue risorse interiori, quelle energie vitali che tutti noi abbiamo e che al momento giusto ci fanno dare il meglio di noi stessi. Una storia che ci racconta come, di fronte a grandi difficoltà, si possono affrontare i problemi nel modo migliore possibile, con il giusto realismo ma anche con l'impetuosa tenacia della speranza.

Mi chiamo Maria Chiara e sono una paziente con reni e fegato policistici e vorrei raccontarvi la mia storia.

Sono nata nel 1960 e all'età di 39 anni sono diventata madre del mio primogenito Francesco, mentre a 41 anni è nata la mia seconda figlia, Caroline. Ora i ragazzi hanno rispettivamente 16 e 15 anni.

Le mie gravidanze sono state serene e senza alcun problema. Insomma, sono diventata madre in maniera totalmente felice e gioiosa.

Dal punto di vista lavorativo ero (e sono?) una "donna in carriera", manager di una importante multinazionale americana, e sono sempre riuscita a conciliare la mia vita professionale con quella familiare, grazie a una accurata organizzazione familiare e naturalmente a degli aiuti: tata, colf ecc. Infatti il mio lavoro mi ha sempre portato a viaggiare molto, soprattutto all'estero.

Insomma, tutto bene.

Nel 2003 (mia figlia aveva appena 1 anno) l'azienda per cui lavoravo, fa fare un check-up a tutti i suoi dirigenti: una giornata intera in una clinica romana a fare esami, ecografie e varie visite specialistiche.

Quando arrivo dall'ecografista, per fare ecografie alla tiroide e all'addome, durante l'esame questo mi dice: «Signora lei lo sa di avere i reni e il fegato policistici?», naturalmente io mi sono presa un colpo: non lo sapevo! All'età di 43 anni, dopo aver fatto tantissime ecografie ginecologiche per le due recentissime gravidanze, ho fatto questa scoperta!

Devo dire che sono andata nel panico! Infatti questo medico mi disse: "Signora, deve immediatamente controllare i suoi figli, fare bucare queste cisti e iniziare immediatamente una dieta a-proteica e senza sale!», insomma mi ha terrorizzato a morte! Ho avuto immediatamente una reazione di panico: mi sono sentita male, sono svenuta, mi hanno dovuto dare delle gocce, mi hanno fatto sdraiare e hanno chiamato mio marito per farmi venire a prendere.

Inutile dirvi che la mia sensazione era che mi fosse caduto

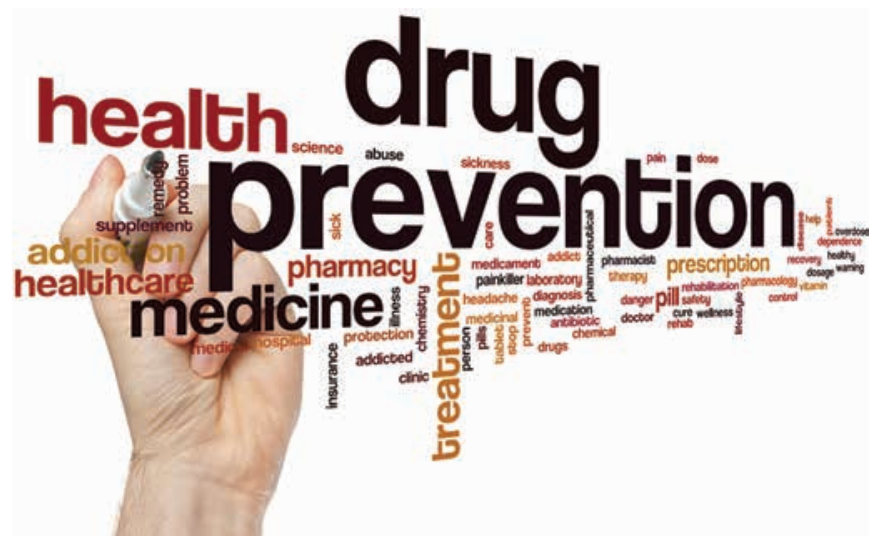

il mondo addosso. Ero soprattutto spaventata per i miei figli e anche per avere davanti a me la visione di una vita in dialisi. La fine insomma!

Uscita dalla clinica, mi sono subito attivata per farmi visitare da uno specialista nefrologo, per sapere di più della malattia e per capire cosa dovevo fare per curarla.

Cosi sono approdata dal medico specialista che mi cura tuttora.

Dalla prima visita ho capito subito due cose: una positiva e una negativa.

La positiva era che, sì avevo molte cisti ma, avendo 43 anni ed essendo del tutto asintomatica (pressione nella norma e filtrato ancora buono), la malattia aveva ancora un decorso lungo davanti e che quindi la mia qualità della vita non sarebbe cambiata sostanzialmente nell'immediato. Avrei dovuto tenere sempre sotto controllo la pressione, bere molta acqua a basso residuo e fare dei controlli periodici. E così è stato, almeno per qualche anno.

La negativa era che ho capito che non c'erano cure. II rene policistico non può essere curato, le cisti ci sono, si riproducono, crescono e la ricerca non aveva (almeno nel 2003) ancora 
trovato dei farmaci validi per bloccare il processo di crescita delle cisti.

Che dire? Era così.

Avevo davanti a me un percorso lungo e la consapevolezza di avere un problema clinico degenerativo non risolvibile: avrei dovuto imparare a convivere con esso, imparando a gestirlo e a vivere al meglio la mia vita.

Qualche anno dopo ho fatto fare le ecografie ai miei figli da cui ho scoperto che purtroppo mia figlia, all'età di 6 anni, già aveva delle cisti, mio figlio invece, per fortuna, ne era immune.

Ora, dopo circa 15 anni, la mia situazione si è evoluta e sono nel cosiddetto stadio IV.

Seguo una dieta a-proteica, prendo vari farmaci per la pressione, ma la mia qualità della vita è ancora decente.

Certo la mia paura per il domani aumenta, non lo nego, ma sono fiduciosa.

E vorrei spiegarvi perché.

Innanzitutto la ricerca sta andando avanti: ci sono numerosi studi su dei farmaci promettenti per la gestione del rene policistico. Questo fatto mi rende più serena nel pensare al futuro di mia figlia. Sicuramente per lei ci saranno delle opportunità di cura. Fantastico!

Per quanto mi riguarda ho preso una importante decisione: mi sto sottoponendo ai controlli per il trapianto pre-emptive, ossia vorrei sottopormi a trapianto (se sarà possibile) prima che la mia insufficienza renale diventi tale da farmi iniziare la dialisi.

Naturalmente è un percorso impegnativo e non facile, ma la prospettiva di poter evitare la dialisi è per me importante.

Questa sensazione di speranza che ho da quando ho intrapreso questa strada, mi ha fatto riflettere sul fatto che a ogni paziente vadano prospettate, da parte dello specialista e al momento giusto, il percorso e le opzioni che ha di fronte, con tutti $\mathrm{i}$ pro e i contro, affinché possa prendere le decisioni migliori per sé e la sua famiglia. La scelta dell'opzione migliore fra la strada del trapianto e la dialisi è soggettiva e va presa in maniera consapevole e con l'adeguato supporto sia medico sia psicologico.

In questo senso vorrei fare un appello ai medici e agli operatori sanitari che seguono i pazienti con APDKD: parlare con i pazienti, dare loro appoggio psicologico, seguirli con umanità e vicinanza, illustrare loro nel dettaglio le opzioni terapeutiche, seguirli insomma dal punto di vista umano è molto importante e fa parte della cura! Impegnatevi in questo senso!

E ai pazienti: chiedete aiuto! Pretendete aiuto dal vostro medico e chiedete il supporto di uno psicologo: il supporto psicologico aiuta veramente a vivere meglio con la malattia. lo l'ho fatto e mi sta aiutando.

Per quanto mi riguarda, in assenza di farmaci al mio stadio di APDKD e considerando che ho dei figli in età adolescenziale, la speranza di riuscire a essere trapiantata prima della dialisi, è l'opzione che mi fa sentire meglio.

Non so se riuscirò in questo, ma la speranza, la prospettiva positiva, è quella che mi fa star meglio adesso.

Poi vedremo.

Auguratemi in bocca al lupo!

Maria Chiara

Published online: May 25, 2017 